\title{
Alimentación del cisne de cuello negro Cygnus melanocoryphus (Aves: Anatidae) en un humedal marino de Chiloé, sur de Chile
}

\section{Feeding of the Black-necked Swan Cygnus melanocoryphus (Birds: Anatidae) in a marine wetland of Chiloe, southern Chile}

\author{
Jaime A. Cursach ${ }^{1,2,3^{*}}$, Jaime R. Rau ${ }^{2}$, Claudio Tobar ${ }^{2,4}$, Jonnathan Vilugrón ${ }^{2} \&$ Lucia E. de la \\ FUENTE $^{5}$ \\ 1Programa de Doctorado en Ciencias mención Conservación y Manejo de Recursos Naturales, Universidad de Los Lagos, \\ Casilla 557, Puerto Montt, Chile. \\ ${ }^{2}$ Laboratorio de Ecología, Departamento de Ciencias Biológicas y Biodiversidad, Universidad de Los Lagos, Casilla 933, \\ Osorno, Chile. \\ ${ }^{3}$ Laboratorio de Investigación Socioambiental, Programa ATLAS, Departamento de Ciencias Sociales, Universidad de Los \\ Lagos, Casilla 933, Osorno, Chile. \\ ${ }^{4}$ Departamento de Ciencias Básicas, Universidad Santo Tomás, Los Carrera 753, Osorno, Chile. \\ ${ }^{5}$ Departamento de Acuicultura y Recursos Agroalimentarios, Universidad de Los Lagos, Casilla 933, Osorno, Chile. \\ *jcurval@gmail.com
}

\section{RESUMEN}

El considerar a Cygnus melanocoryphus como un herbívoro generalista que se alimenta de las algas y pastos marinos más abundantes en los humedales - entre ellos los costeros - que habita, permite suponer la existencia de interacciones aún no reportadas entre esta ave acuática y los cultivadores de algas marinas del sur de Chile, por ello el objetivo de este estudio fue conocer la alimentación de C. melanocoryphus en un humedal marino del sur de Chile, en donde tradicionalmente se ha realizado el cultivo del alga pelillo (Gracillaria chilensis). El trabajo se desarrolló durante el

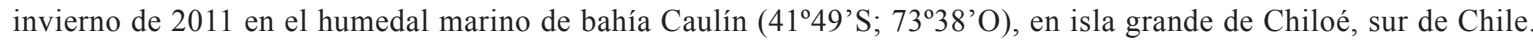
C. melanocoryphus destinó la mayor parte de su tiempo a la alimentación, observándose en esta ave una conducta alimenticia de herbívoro selectivo hacia el consumo no estricto pero preferente del alga Ulva taeniata. El ciclo de marea afectó la distribución espacial de C. melanocoryphus; durante los períodos de marea baja éstos se concentraron en la zona intermareal alimentándose de las frondas de $U$. taeniata retenidas o asentadas en los sistemas de cultivo de G. chilensis. Mientras que durante los períodos de marea alta C. melanocoryphus ingresó a la zona supramareal siguiendo las frondas de U. taeniata en la deriva de las corrientes, observándolos alimentarse también de los pastos salados (Distichlis spicata y Selliera radicans) dominantes en la zona estuarina (fuera del agua). El conocimiento obtenido permite proponer la hipótesis de un servicio ambiental brindado por C. melanocoryphus a los algueros del humedal, mediante la remoción del alga U. taeniata desde los sistemas de cultivo de G. chilensis liberando su competencia por sustrato y luz para crecer.

Palabras clave: acuicultura, herbivoría, liberación competitiva, servicio ambiental.

\begin{abstract}
Considering Cygnus melanocoryphus as a generalist herbivore that feeds on algae and seagrass more abundant in wetlands, suggests the existence of unknown interactions between this waterfowl and the seaweed farmers in Southern Chile. Therefore the aim of this work was study the feeding of $C$. melanocoryphus in a marine wetland of southern Chile, where traditionally it has made the cultivation of algae Gracillaria chilensis. This work was conducted during the winter of 2011 in the marine wetland of Caulin bay $\left(41^{\circ} 49^{\prime} \mathrm{S} ; 7^{\circ} 38^{\prime} \mathrm{W}\right)$, in the Island of Chiloé, Southern Chile. $C$. melanocoryphus spent most of their time feeding, observing in this bird a behavior of selective herbivory food towards preferential but not exclusive consumption of the seaweed Ulva taeniata. The tidal cycle affected the spatial distribution of C. melanocoryphus; during times of low tide these were concentrated in the intertidal zone feeding on the fronds of $U$. taeniata retained or settled in the farming systems of $G$. chilensis. While during high tide periods $C$. melanocoryphus entered to the supratidal zone by following the fronds of $U$. taeniata in the drift of the currents, watching they feed on the dominant seagrass (Distichlis spicata and Selliera radicans) in the estuarine zone. The knowledge obtained allows
\end{abstract}


argue the hypothesis of an environmental service provided by C. melanocoryphus for the "algueros" of the wetland, by removing of the seaweed $U$. taeniata from culture systems of $G$. chilensis freeing his competition over substrate and light to grow.

KEYWORDS: aquaculture, competitive release, herbivory, environmental service.

\section{INTRODUCCIÓN}

El cisne de cuello negro Cygnus melanocoryphus (Molina, 1782) (en adelante CCN) es un ave acuática perteneciente a la familia Anatidae y único representante del género Cygnus en América del Sur (Schlatter et al. 1991b). Su distribución geográfica se amplía desde el sur de Brasil y norte de Chile hasta la península Antártica, abarcando toda Patagonia e islas Malvinas (BirdLife International 2012). El criterio internacional de conservación considera al CCN como una especie de Preocupación Menor (LC) (BirdLife International 2012), mientras que a nivel local en Chile está categorizada como en peligro de extinción desde la región del Bío-Bío a la región de Los Lagos (SAG 2011).

El conocimiento de la alimentación del CCN en Chile corresponde principalmente a su estudio en ambientes dulceacuícolas, donde se alimenta casi exclusivamente de la planta acuática invasora Egeria densa (Schlatter et al. 1991a, Corti \& Schlatter 2002). Los escasos reportes sobre la dieta del $\mathrm{CCN}$ en ambientes marinos señalan el consumo de algas marinas verdes como Ulva lactuca (Linnaeus) y el pasto marino Ruppia marítima (Linnaeus, 1753), especies consideradas como de amplia dispersión y tolerancia a la contaminación del sistema litoral, señalando un patrón de eutroficación en sus sitios de alimentación (Schlatter et al. 1991b, Corti 1996, Bortolus et al. 1998, Schlatter 2005). En general, el estado del conocimiento del CCN lo considera como un consumidor primario oportunista que utiliza los recursos del ambiente que se encuentran en mayor abundancia y de más fácil acceso (Corti 1996, Corti \& Schlatter 2002, Figueroa-Fábregas et al. 2006).

El considerar al CCN como un ave herbívora consumidora de las plantas acuáticas más abundantes de los sistemas que habita, despierta el cuestionamiento sobre potenciales interacciones entre esta especie y los cultivadores de algas marinas del sur de Chile (i.e., archipiélago de Chiloé) teniendo en cuenta la inmigración de $\mathrm{CCN}$ ocurrida a la zona tras el "Desastre del río Cruces", en las cercanías de Valdivia (Muñoz-Pedreros 2004, Jaramillo et al. 2007, Sepúlveda \& Villarroel $\left.2005^{1}\right)$. El archipiélago de Chiloé $\left(42-43{ }^{\circ} \mathrm{S}\right)$ se compone de numerosas islas, mares interiores, canales, estuarios y humedales marinos, con un amplio régimen de marea que son utilizados por las comunidades locales que extraen y cultivan de forma artesanal el alga pelillo (Gracillaria chilensis CJ Bird, McLachlan \& EC Oliveira)

\footnotetext{
1 http://www.derechoalagua.cl/mapa-de-conflictos/desastre-
} ecologico-de-celco-en-el-santuario-rio-cruces/ en la zona intermareal (Hucke-Gaete et al. 2010). La presencia del CCN (i.e., herbívoro oportunista) en zonas intermareales dominadas por el cultivo de algas marinas (i.e., pelillo), hace suponer la ocurrencia de interacciones entre esta ave acuática y los pescadores artesanales dedicados a dicho cultivo. Por lo anterior, el objetivo de este trabajo fue conocer la alimentación del $\mathrm{CCN}$ en un humedal marino de la isla grande de Chiloé donde tradicionalmente se ha cultivado el alga pelillo, estudiando la disponibilidad de algas para consumo, sus contenidos nutricionales y palatabilidad, junto con la conducta y selectividad alimenticia de esta ave acuática.

\section{MATERIALES Y MÉTODOS}

\section{Sitio DE ESTUdio}

El estudio fue realizado en el humedal marino de bahía Caulín (41 $49^{\circ} \mathrm{S}$; $\left.73^{\circ} 38^{\prime} \mathrm{O}\right)$, ubicado en la comuna de Ancud, isla grande de Chiloé, sur de Chile (Figura 1). Dicho humedal marino posee una superficie de $435 \mathrm{ha}$, con sustrato principalmente arenoso y con la importante presencia del estuario del río Huenque junto a varios otros esteros que desembocan en la bahía, destacando la extensa amplitud mareal que posee la zona intermareal. En general, el lugar se caracteriza por presentar abundantes lluvias (4000 a 7000 $\mathrm{mm}$ por año) y una temperatura ambiente promedio de $11^{\circ}$ C (Hucke-Gaete et al. 2010).

El humedal marino de bahía Caulín es considerado un sitio de importancia para la observación y conservación de aves acuáticas a nivel nacional (i.e., zona prohibida de caza) e internacional (i.e., sitio AICAS) (Ortiz et al. 2009, SAG 2011). Sus principales actividades productivas tienen relación con la extracción y recolección de mariscos y algas. En aproximadamente 65 ha de la zona intermareal se cultiva artesanalmente el alga pelillo por alrededor de 225 personas (Andres et al. 2009). El pelillo cultivado es plantado en el sustrato intermareal de forma indirecta a través de cuerdas instaladas mediante estacas de madera. En dichas cuerdas (de polipropileno con longitudes de $20 \mathrm{~m}$ ) se adhieren previamente las plantas de pelillo y luego son instaladas en el intermareal, donde el alga crece mediante las condiciones naturales del entorno.

\section{OBTENCIÓN DE LOS DATOS}

Para las aves del género Cygnus y en especial para el $\mathrm{CCN}$, se ha descrito que la época invernal constituye una 
temporada alimenticia crítica cuando los requerimientos nutricionales son mayores, debido a que estas aves se preparan para comenzar la época reproductiva y la posterior crianza y desarrollo de sus polluelos durante la primavera (Scott \& The Wildfowl Trust 1972, Corti 1996). Por ello, durante junio a septiembre (temporada invernal) 2011 se estudió la ecología trófica del CCN en el humedal marino de bahía Caulín, Chiloé, mediante observaciones conductuales directas, experimentos de campo y análisis de sus heces. Dentro de la bahía, el esfuerzo de estudio se enfocó en el área central del humedal marino abarcando una superficie aproximada de 161 ha.

Se realizaron 13 sesiones de observación sobre la conducta del $\mathrm{CCN}$, las cuales consistieron en un monitoreo continuo de la actividad realizada por uno o dos individuos adultos durante un tiempo de 20 minutos (Altmann 1973), apoyando las observaciones mediante lentes binoculares (16 x 50) y telescopio $(40 \times 60)$. Las sesiones fueron realizadas en diferentes zonas del humedal marino, durante horarios de mañana $(08: 00-10: 00$ h), medio día $(13: 00-15: 00$ h) y tarde $(17: 00-19: 00 \mathrm{~h})$.

Se recolectó un total de 40 heces del CCN durante diferentes días dentro del humedal. Comúnmente, los $\mathrm{CCN}$ defecan en el agua, pero ocasionalmente sus heces (de forma tubular, contextura gelatinosa y para este caso con intensa coloración verde) pueden ser encontradas sobre la línea de marea en las playas, permitiendo ser recolectadas (Figura 2). Las muestras (cada una de las heces) fueron analizadas mediante la técnica microhistológica descrita por Johnson et al. (1983). Esta técnica se basa en la comparación de estructuras de células vegetales y sus diferentes patrones persistentes más o menos intactos en las heces con una colección de referencia, alcanzándose la identificación a nivel de especies (Owen 1975, Johnson et al. 1983, Corti \& Schlatter 2002). Cinco campos microscópicos fueron observados en cada muestra con un aumento óptico de 100 x (totalizando 200 campos microscópicos).

Para conocer la oferta de algas disponibles (para consumo del $\mathrm{CCN}$ ) en la planicie intermareal del humedal se utilizaron 11 transectos de $100 \mathrm{~m}$ cada uno, de forma perpendicular a la línea de marea y distantes a $200 \mathrm{~m}$ entre sí. En cada transecto se registró la riqueza y cobertura (\%) de las macroalgas presentes mediante un cuadrante de 0,25 $\mathrm{m}^{2}$, el cual fue lanzado repetitivamente cada $20 \mathrm{~m}$ a través del transecto. En esta actividad, se recolectaron muestras de cada especie de plantas acuáticas encontradas en el sitio de estudio, las cuales posteriormente permitieron estudiar su composición físico-química, así como también para preparar una colección de referencia para el análisis de las heces del CCN.

Para cada especie de planta acuática colectada en el sitio de trabajo, se estudió su composición nutricional mediante análisis de humedad, proteínas, cenizas, fibra y extracto etéreo, según las metodologías utilizadas por el CEALUlagos (<http://www.ceal.ulagos.cl $>$ ). Mientras que de forma paralela, para estas mismas especies se estudió su textura mediante mediciones de dureza, adhesividad y elasticidad en sus tejidos, utilizando para ello el instrumento LFRA Texture Analyzer (Brookfield Texture Pro Lite).

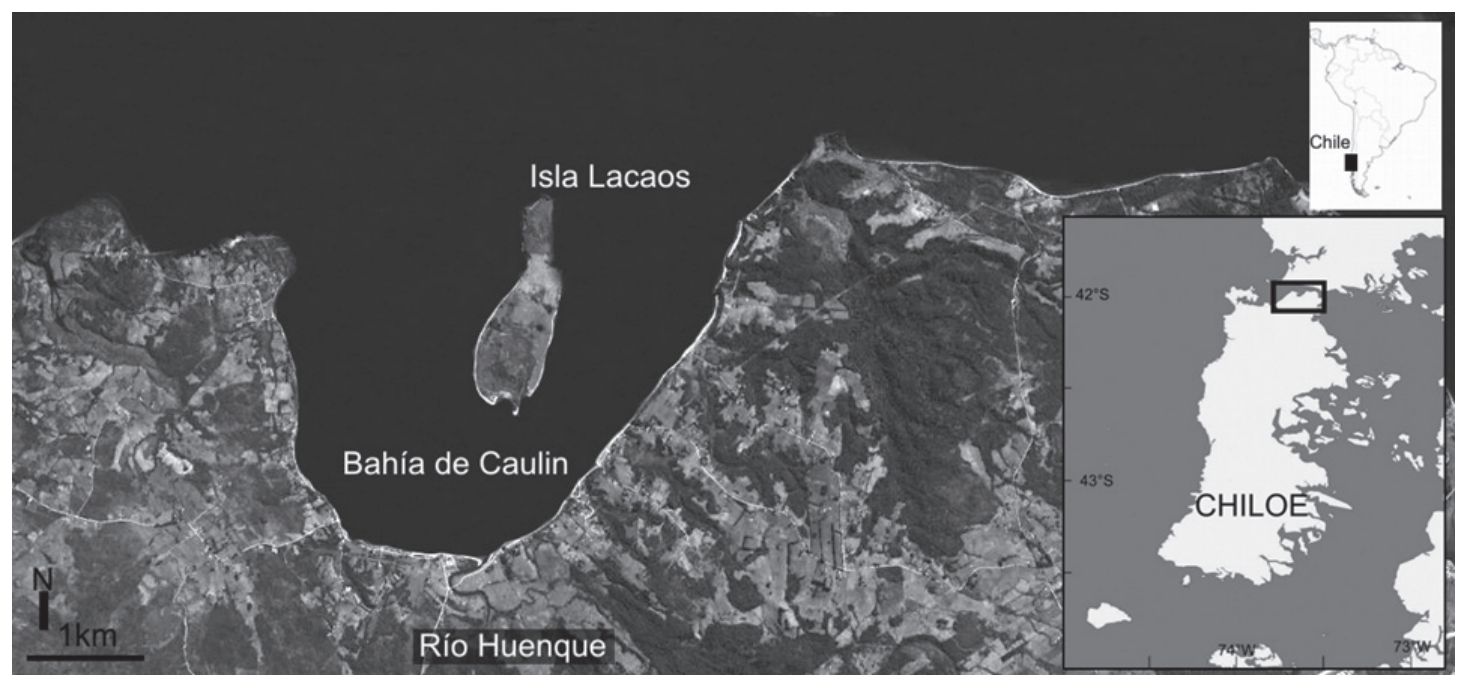

Figura 1. Ubicación geográfica del humedal marino de bahía Caulín, isla grande de Chiloé, sur de Chile.

FIGURE 1. Geographic location of the Caulín Bay marine wetland, big island of Chiloé, Southern Chile. 
Para determinar la existencia de selectividad en la conducta alimenticia del CCN se instaló un total de ocho líneas experimentales en diferentes días y sectores del humedal. Cada línea consistió en una cuerda $(1 \mathrm{~mm} ø)$ de $3 \mathrm{~m}$ de longitud en la cual se adosaron dos ramilletes de cada una de las cuatro especies de plantas que conformaron la oferta de algas para $\mathrm{CCN}$ en el sitio de estudio. Así y durante los períodos de marea baja se adosó cada línea experimental en la planicie intermareal del humedal, quedando sumergidas en los momentos de marea alta, tiempo en el cual los CCN se acercaron a alimentarse de las plantas adosadas (Figura 3 ). Cada ramillete de plantas fue pesado antes y después de cada experiencia (peso húmedo), para así poder comparar el consumo de cada especie por parte de los CCN.

En forma complementaria, se realizaron entrevistas semiestructuradas (Briones 1998, Huntington 1998) a los habitantes de bahía Caulín ( $\mathrm{n}=15$; con edades desde 35 a 72 años) encontrados en el humedal marino, en relación al conocimiento ecológico local sobre la alimentación de los $\mathrm{CCN}$ en el lugar (i.e., ¿qué comen y dónde?, ¿desde cuándo?, ¿cómo era antes?).

ANÁLISIS DE LOS DATOS

Durante las sesiones de observación etológica del CCN se identificaron seis categorías conductuales (Altmann 1973,
Corti 1996), consistentes en: 1) Acicalamiento: se realiza con el pico por sobre todo el plumaje, también abriendo y sacudiendo las alas, moviendo la cola hacia los costados rápidamente $\mathrm{y}$ con el cuello levemente estirado. 2) Alimentación: consistió en tres posturas relacionadas con la obtención de sus presas algales; a) el CCN come en la superficie del agua sin efectuar ningún tipo de inmersión, alimentándose de plantas sumergidas a poca profundidad o restos de las mismas en la superficie. b) El CCN hunde la totalidad del cuello bajo el agua para alcanzar plantas acuáticas totalmente sumergidas. c) El $\mathrm{CCN}$ hunde todo el cuello y verticaliza la mitad anterior del cuerpo en el agua, dejando fuera la porción caudal del mismo, alcanzando las plantas acuáticas sumergidas a una mayor profundidad. 3) Beber agua dulce: mediante el deslizamiento de su pico ingiere agua dulce presente en la superficie del mar (i.e., por diferencia de densidades), en zonas de estuario o en los abundantes desagües de esteros en la playa. 4) Búsqueda de alimento: mediante breves desplazamientos en nado, el CCN mueve su cuello en la superficie buscando alimento. 5) Nadar: esta conducta se define al observar al CCN en la superficie del agua sin efectuar otra actividad que no sea su desplazamiento en nado. 6) Vocalización: emisión de silbidos y graznidos durante momentos de socialización, estando relativamente estáticos en el agua.

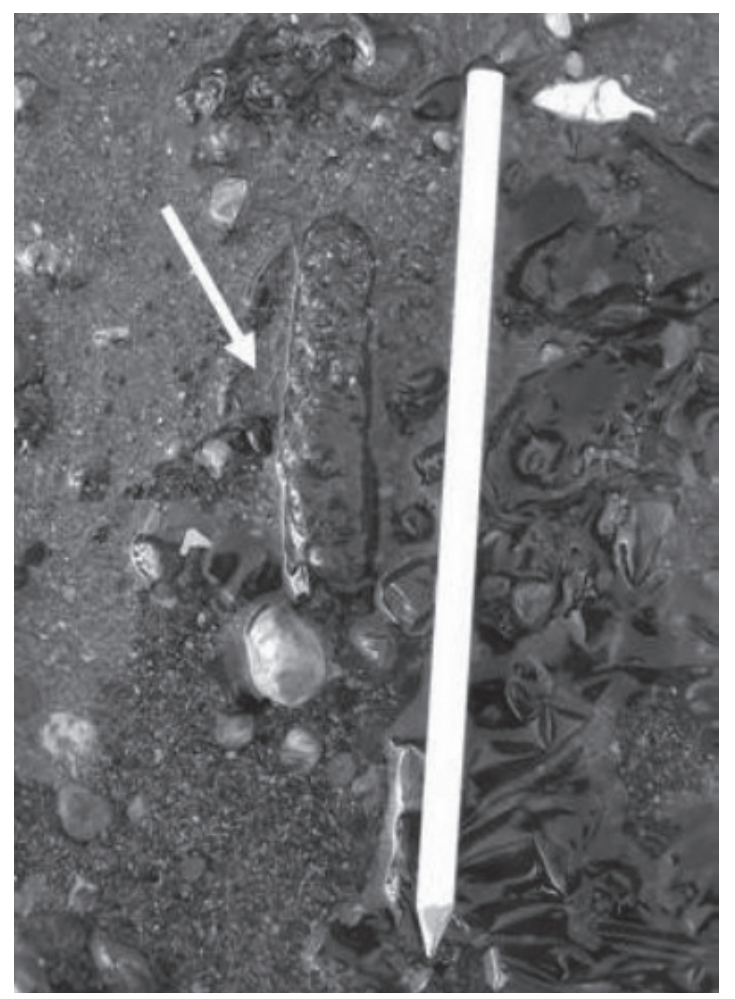

FIGURA 2. Fotografía de una hez de C. melanocoryphus encontrada en la zona intermareal del humedal marino de bahía Caulín, sur de Chile.

FIGURE 2. Photograph of a C. melanocoryphus faeces found in the Caulín Bay marine wetland, Southern Chile. 

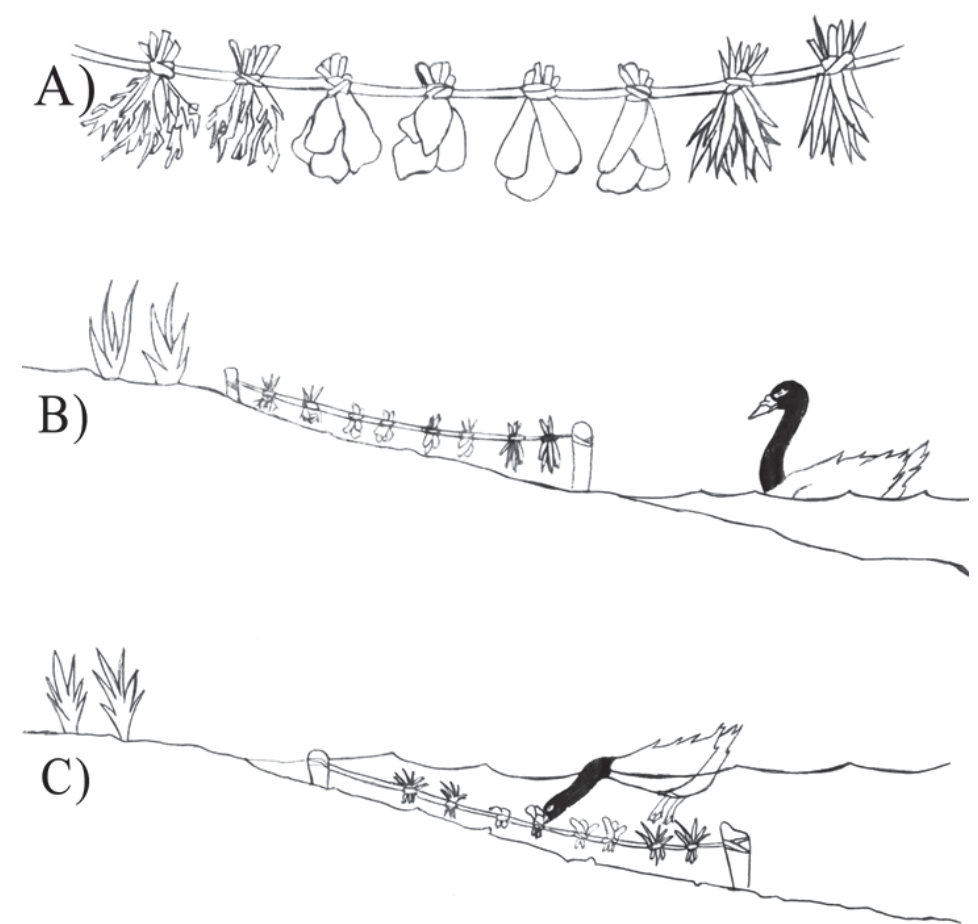

FIgURA 3. Esquema de una línea experimental (A) utilizada para el estudio de selectividad alimenticia de C. melanocoryhus, en el humedal marino de bahía Caulín, sur de Chile. Donde, B: instalación de una línea experimental durante marea baja, y C: alimentación de $C$. melanocoryphus en la línea experimental durante marea alta.

Figure 3. Schematic of an experimental line (A) used to study feeding selectivity of C. melanocoryphus in the marine wetland of Caulín Bay, Southern Chile. Where, B: installation of an experimental line during low tide, and $\mathbf{C}$ : feeding of C. melanocoryphus in the experimental line during high tide.

Para determinar diferencias estadísticas en los tiempos destinados por los $\mathrm{CCN}$ a cada una de las categorías conductuales, se utilizó la prueba de Kruskal-Wallis $(\alpha=$ 0,05) (Zar 1999). La misma prueba estadística fue utilizada para determinar diferencias en los tiempos conductuales de los CCN para los diferentes horarios del día (mañana, medio día y tarde) en que se realizaron las observaciones. Por otra parte, se realizaron comparaciones en los tiempos destinados por los $\mathrm{CCN}$ a cada una de las categorías conductuales para cada zona de observación durante los distintos períodos del ciclo mareal (marea baja y marea alta), utilizando la prueba U de Mann-Whitney $(\alpha=0,05)$ (Zar 1999).

A partir del análisis de heces del CCN, se obtuvo la distribución de frecuencias relativas de los ítems dietarios (Zar 1999) de acuerdo a la presencia de células de plantas observadas en los campos microscópicos (Johnson et al. 1983). Los resultados fueron expresados como porcentajes de ocurrencia de las células de cada especie de planta para el total de campos microscópicos observados.

Para determinar diferencias estadísticas en el consumo de los CCN sobre una u otra alga (mediante el peso de cada ramillete de plantas, antes y después de la experiencia) en los experimentos de selectividad alimenticia, se utilizaron pruebas pareadas de Wilcoxon $(\alpha=0,05)$ (Zar 1999). La texturometría de las potenciales plantas acuáticas consumidas por el CCN en bahía Caulín, permitió conocer el grado de dureza, adhesividad y elasticidad de cada planta. Para determinar diferencias estadísticas en dichos componentes para todas las especies algales estudiadas, se utilizó la prueba de Kruskal-Wallis $(\alpha=0,05)$ (Zar 1999).

\section{RESULTADOS}

De la totalidad de observaciones sobre la conducta del $\mathrm{CCN}$, la mayor parte del tiempo (Chi-cuadrado $=44,06$; g.l. $=5 ; \mathrm{P}=0,0001)$ fue observado alimentándose $(57,40$ $\%$ ) de las frondas de "lamilla" Ulva taeniata (Setchell \& NL Gardner), seguido por el tiempo destinado (20,70 \%) a movilizarse (mediante natación) dentro de la bahía y para beber el agua dulce $(7,10 \%)$ que cae del río y esteros (Tabla 1). No existieron diferencias significativas en el tiempo conductual de los CCN durante los diferentes períodos del ciclo mareal (Tabla 1). 
El ciclo mareal afectó la distribución espacial de los CCN dentro de la bahía. Durante los períodos de marea alta los $\mathrm{CCN}$ se concentraron principalmente $(\mathrm{Z}$ ajustado $=1,99$; $\mathrm{P}=0,04)$ en el río Huenque, adentrándose en sus aguas por beneficio de la altura de marea, observándolos alimentarse de las lamillas arrastradas por la marea (junto con beber el agua dulce del río), así como también alimentándose de la asociación vegetal de pastos salobres [i.e., el pasto salado (Distichlis spicata LE Greene) y la maleza de marisma (Selliera radicans Cav)] dominantes en la zona supramareal de la marisma. Durante la marea baja los $\mathrm{CCN}$ se concentraron principalmente $(\mathrm{Z}$ ajustado $=-1,99$; $\mathrm{P}=0,04)$ en el sector donde se ubica el cultivo del pelillo, observándolos alimentarse de las frondas de lamillas retenidas o asentadas en los sistemas de cultivo (i.e., varas de madera y cuerdas de polietileno).

A partir del análisis de las heces del CCN $(n=40)$, se identificó a la macroalga lamilla como el principal ítem alimenticio del $\mathrm{CCN}$ en el área de estudio, mientras que el pasto salado fue la segunda planta con mayor importancia en su dieta (Tabla 2).

Con respecto a la oferta de algas para el consumo del CCN en el humedal marino de bahía Caulín, el estudio del ensamble de macroalgas presentes en la zona intermareal indicó una riqueza compuesta por tres especies algales, de las cuales la mayor abundancia correspondió al pelillo $(94,70 \%)$, seguido en menor proporción por las algas lamilla $(4,80 \%)$ y Codium fragile (Suringar) Hariot $(0,40 \%)$. Pese a que no se registraron plantas del alga luga Gigartina skottsbergii (Setchell \& NL Gardner, 1936) creciendo en la zona intermareal, esta especie fue comúnmente observada varada en la playa del humedal marino estudiado.
Sin haber sido evaluada de forma cuantitativa, se observó una gran biomasa de lamillas varadas en la playa por efecto de las corrientes, las cuales provenían de los abundantes bancos naturales existentes en el canal de Chacao (registro originado a partir del Conocimiento ecológico local).

Los experimentos de selectividad dietaria $(\mathrm{n}=8)$ indicaron que los CCN se alimentaron principal y significativamente $(\mathrm{Z}=2,52$; g.l. $=7 ; \mathrm{P}=0,01)$ del alga lamilla (Tabla 3$)$. Esta alga presentó importantes concentraciones de proteína y un alto porcentaje de fibra en su composición, con respecto a las plantas posibles de ser consumidas por el $\mathrm{CCN}$ en el sitio de estudio (Tabla 4).

Las mediciones de textura en las cuatro especies de plantas que conforman la oferta alimenticia potencial del CCN en el sitio de estudio indicaron que el pasto salado posee los mayores valores de dureza (Chi-cuadrado $=0,86$; g.l. $=3$; $\mathrm{P}=0,01)$ y elasticidad (Chi-cuadrado $=8,80 ;$ g.l. $=3 ; \mathrm{P}$ $=0,03)$. Mientras que sin presentar significancia, el alga lamilla exhibió la menor adhesividad (Tabla 5).

De la información obtenida a través de las entrevistas a los(as) habitantes de bahía Caulín, en relación al conocimiento ecológico local sobre la ecología trófica del $\mathrm{CCN}$ en dicho humedal, ésta indicó que los CCN siempre han sido observados alimentándose en el lugar, casi exclusivamente del alga lamilla que deriva con las corrientes de marea, siempre buscando las frondas más frescas, blandas y de intensa coloración verde. Ellos(as) argumentaron que la elección de lamilla por sobre las otras macroalgas presentes se debe a que esta es más blanda y fácil de digerir. Dos entrevistados indicaron la posible alimentación del CCN sobre pequeños invertebrados presentes tanto en la playa como entre las plantas de pelillo cultivado.

TABla 1. Tiempo conductual promedio (porcentaje) observado en C. melanocoryphus a diferentes horarios del día y etapas del ciclo mareal en bahía Caulín, Chiloé, sur de Chile.

TABLE 1. Average behavioral time (percentage) observed on C. melanocoryphus at different day schedules and tide cycles stages in Caulin Bay, Chiloe, Southern Chile.

\begin{tabular}{lrrrrrrr}
\hline \multirow{2}{*}{ Conducta } & \multirow{2}{*}{ Total } & \multicolumn{2}{c}{ Ciclo de marea } & \multicolumn{3}{c}{ Horario } \\
\cline { 3 - 7 } & & Baja & Alta & Mañana & Medio día & TARde \\
\hline Acicalamiento & 5,1 & 6,3 & 4,1 & 7,4 & 3,5 & 2,5 \\
Bebiendo agua & 7,1 & 6,4 & 8,2 & 5,8 & 3,5 & 11,5 \\
Búsqueda de alimento & 4,6 & 3,2 & 5,1 & 4,9 & 7,1 & 2,7 \\
Alimentación & 57,4 & 58,2 & 56,1 & 53,7 & 57,8 & 62,8 \\
Vocalización & 5,1 & 5,7 & 5,1 & 4,9 & 5,4 & 5,2 \\
Nadando & 20,7 & 20,2 & 21,4 & 23,3 & 22,7 & 15,3 \\
\hline
\end{tabular}


Dieta de Cygnus melanocoryphus en humedal marino de Chiloé: JAIME A. CurSACH ET AL.

TABLA 2. Análisis microhistológico de las heces de C. melanocoryphus en el humedal marino de bahía Caulín ( $\mathrm{n}=200$ campos microscópicos), Chiloé, sur de Chile. Los resultados indican la frecuencia relativa de células de cada planta, expresados como porcentaje de la ocurrencia de especies de plantas en el total de campos microscópicos observados.

TABLE 2. Microhistological analysis of the C. melanocoryphus faeces in the marine wetland of Caulin Bay ( $\mathrm{n}=200$ microscopic fields), Chiloe, southern Chile. The results indicate the relative frequency of each plant cells, expressed as a percentage of the occurrence of plant species in total observed microscopic fields.

\begin{tabular}{lcc}
\hline EsPECIES DE PLANTAS & $\begin{array}{c}\text { FRECUENCIA } \\
\text { RELATIVA }\end{array}$ & $\begin{array}{c}\% \text { DE } \\
\text { OCURRENCIA }\end{array}$ \\
\hline Ulva taeniata & 57386 & 95,58 \\
Distichlis spicata & 2180 & 3,63 \\
Gracillaria chilensis & 459 & 0,76 \\
Gigartina skottsbergii & 14 & 0,02 \\
\hline
\end{tabular}

Tabla 3. Porcentaje promedio del consumo (con rangos mín. y máx.) de plantas acuáticas de C. melanocoryphus, en experimentos de selectividad alimenticia realizados en el humedal marino de bahía Caulín, Chiloé.

TABLE 3. Average consumption percentage (with ranges min. and max.) of aquatic plants by C. melanocoryphus during feeding selectivity experiments performed in the marine wetland of Caulin Bay, Chiloe.

\begin{tabular}{lc}
\hline ESPECIES DE PLANTAS & $\%$ DE CONSUMO \\
\hline Ulva taeniata & $73,11 \pm 21,97(35,57$ a 97,54$)$ \\
Distichlis spicata & $10,62 \pm 7,88(1,57$ a 27,79$)$ \\
Gracillaria chilensis & $2,18 \pm 4,27(0$ a 11,3$)$ \\
Gigartina skottsbergii & sin consumo \\
\hline
\end{tabular}

TABLA 4. Composición nutricional (en porcentaje de materia seca) de las cuatro especies de plantas que conforman la oferta alimenticia de C. melanocoryphus en el humedal marino de bahía Caulín, Chiloé, sur de Chile. La materia seca fue obtenida como diferencia del porcentaje de humedad registrado en cada planta. E.N.N.: Extracto No Nitrogenado.

TABLE 4. Nutritional composition (percentage of dry matter) of the four plant species that make up the plant food availability of $C$. melanocoryphus in the marine wetland of Caulin Bay, Chiloe, southern Chile. The dry matter was obtained as the difference of the percentage of moisture recorded for each plant. E.N.N.: Nitrogen-free extract.

\begin{tabular}{lcccc}
\hline Ítem NUTRICIONAL & $\begin{array}{c}\text { Distichlis } \\
\text { spicata }\end{array}$ & $\begin{array}{c}\text { Gigartina } \\
\text { skottsbergii } \\
(\%)\end{array}$ & $\begin{array}{c}\text { Ulva taeniata } \\
(\%)\end{array}$ & $\begin{array}{c}\text { Gracillaria } \\
\text { chilensis } \\
(\%)\end{array}$ \\
\hline Ceniza & 15,67 & 27,53 & 30,24 & 32,88 \\
Proteína & 11,53 & 18,14 & 21,29 & 21,89 \\
Materia grasa & 2,36 & 0,69 & 1,29 & 0,98 \\
Fibra & 16,85 & 0,69 & 41,89 & 17,86 \\
E.N.N. & 53,59 & 52,95 & 5,29 & 26,39 \\
\hline
\end{tabular}


TABLA 5. Valores promedios de la texturometría (con rangos mín. y máx.) realizada a las plantas acuáticas que compusieron la oferta de presas para C. melanocoryphus, en el humedal marino de bahía Caulín, Chiloé.

TABLE 5. Average texturometric values (with ranges min. and max.) of aquatic plants that composed the food offer of C. melanocoryphus, in the marine wetland of Caulin Bay, Chiloe.

\begin{tabular}{lcrlrl} 
Texturometría & \multicolumn{1}{c}{ Distichlis spicata } & Gigartina skottsbergii & Ulva taeniata & Gracillaria chilensis \\
\hline Dureza (g) & $18 \pm 4,95(12,2$ a 24,6$)$ & $9 \pm 1,88(6,8$ a 11,6$)$ & $10,72 \pm 1,29(9$ a 12,2$)$ & $11,24 \pm 1,85(8,8$ a 13,2$)$ \\
& & & & & \\
Adhesividad (g.s) & $-0,01 \pm 0,02(-0,06$ a 0$)$ & $-0,058 \pm 0,09(-0,22$ a 0$)$ & $-0,18 \pm 0,27(-0,67$ a 0$)$ & $0 \pm 0(0$ a 0$)$ \\
& & & &
\end{tabular}

\section{DISCUSIÓN}

La alimentación del CCN en el humedal marino de bahía Caulín estuvo compuesta principalmente por lamilla, alga marina cuya composición es similar (i.e., alga verde) a las plantas acuáticas reportadas como su alimento en el ambiente marino y dulceacuícola (Corti 1996, Bortolus et al. 1998, Corti \& Schlatter 2002).

Entre las interacciones involucradas en la estructuración de los diversos ecosistemas, la herbivoría es considerada como una de las más importantes, debido a que constituye la base de las cadenas tróficas, y por ende, condiciona el flujo de materia y energía a través de las comunidades (Cáceres \& Ojeda 2000). Varios factores han sido propuestos como importantes en los procesos de búsqueda, selección y consumo de un determinado ítem alimenticio para un herbívoro, entre los más recurrentes destacan: el valor energético de la planta, la eficiencia con que los organismos digieren los diferentes tipos de plantas, la abundancia relativa, contenido proteico de la planta, su forma y textura (Lobel 1981). Para el caso del humedal marino de bahía Caulín, dentro de la oferta de algas para el consumo del $\mathrm{CCN}$, el alga lamilla presentó baja dureza y adhesividad en su textura, con altos contenidos de fibra y proteína en su composición. Estas razones parecen suficientes para explicar la conducta alimenticia selectiva del CCN sobre el alga lamilla, aun así, se ha descrito que el consumo preferencial de algas verdes por parte de peces herbívoros en la costa de Chile se correlacionaría más con la probable ausencia de compuestos "anti-herbívoros" presentes en dichas algas, que con las características físico-químicas de las algas verdes en relación a las algas rojas y pardas presentes en el medio (Cáceres \& Ojeda 2000), situación que requiere de futura investigación para esclarecer su influencia en la conducta alimenticia de aves acuáticas como el CCN.

El conocimiento ecológico local sobre la alimentación del CCN en bahía Caulín coincide con variados puntos de la información científica obtenida por el presente trabajo, salvo en la percepción de su potencial depredación sobre meiofauna. Existen reportes sobre el consumo eventual de materias animales por parte de los CCN (Johnson \& Goodall 1965, Rau 1980) que no deberían ser obviados por los ornitólogos, teniendo en cuenta que aves acuáticas consideradas comúnmente como herbívoras [i.e., tagüa común Fulica armillata (Vieillot, 1817)] han presentado recientes innovaciones en su dieta, depredando sobre macroinvertebrados [i.e., el cangrejo Cyrtograpsus angulatus (Dana, 1851)] en ambientes costeros de Argentina (García et al. 2008). Por estas razones se requieren de continuas observaciones sobre la alimentación del CCN ya que su conducta alimenticia puede ser influenciada por procesos socio-ecológicos aún desconocidos, como por ejemplo alteraciones del hábitat o cambios en la disponibilidad y consumo de energía por parte de las aves.

Una de las variables ambientales identificadas como influyentes en la ecología trófica del CCN es el nivel o profundidad de la columna de agua, la que afecta en sus posturas de alimentación, distribución espacial y abundancia (Schlatter et al. 2002). Para el caso de un humedal marino del sur de Chile, el ciclo de marea determina el nivel y cobertura de la masa de agua en estos sistemas, influyendo en la distribución y abundancia del CCN (Cursach et al. 2010). En el humedal marino de bahía Caulín, el ciclo de marea afectó la distribución espacial del CCN dentro de la bahía, ya que durante los períodos de marea baja éstos se 
concentraron en la zona intermareal alimentándose de las frondas de lamilla retenidas o asentadas en los sistemas de cultivo del pelillo. Mientras que durante los períodos de marea alta los CCN se concentraron principalmente en el río Huenque, adentrándose en sus aguas por beneficio de la altura de marea, observándolos alimentarse de los pastos salados dominantes en la zona supramareal del estuario. Una conducta similar a esta última, fue la observada en el cisne trompetista Cygnus buccinator (Richardson, 1832) en Columbia Británica (Canadá), el cual durante los períodos de marea alta salió del agua para alimentarse en praderas cultivadas de la zona supramareal del estuario estudiado (McKelvey \& Verbeek 1988).

Es importante destacar que el CCN no posee las características fisiológicas propias de un ave marina (ver Schlatter \& Simeone 1999), por lo que sus restricciones fisiológicas al ambiente marino pueden ser disminuidas mediante el consumo de agua dulce, de forma similar a lo realizado por otras aves no marinas que habitan el ambiente costero (Sabat et al. 2006). Así, la disponibilidad de agua dulce puede ser la respuesta a la razón por la cual las mayores concentraciones de $\mathrm{CCN}$ registradas en el ambiente marino de Chile han sido reportadas para las costas del sur del país (Markham 1971, Rau 1980, Vuilleumier 1997, Gibbons et al. 2007, Matus et al. 2010); ecosistema caracterizado por estar conformado por canales, fiordos y abundantes desembocaduras de ríos que facilitan la presencia de hábitats con menores concentraciones de salinidad (Hucke-Gaete et al. 2010).

El alga lamilla consumida por el $\mathrm{CCN}$ fue obtenida principalmente mediante frondas presentes a la deriva de las corrientes de marea, así como también mediante el consumo de las plantas asentadas en los sistemas de cultivo del pelillo. Una evaluación somera del nivel de asentamiento de lamillas en las líneas de cultivo del pelillo en bahía Caulín, indicó una media de 14,6\% (con rangos desde 0 a 42,1\%; $\mathrm{n}=6$ ) de cobertura de lamillas por línea. El alga lamilla es dependiente de sustratos duros para su asentamiento y desarrollo (Hoffmann \& Santelices 1997), logrando crecer sobre los sistemas de cultivo artesanal del pelillo en bahía Caulín. En dicho lugar, las lamillas desarrollan grandes frondas que con el movimiento del agua logran desprender al pelillo cultivado (J. Cursach, obs. pers.), proponiéndose así una interacción competitiva por sustrato y captación de luz entre ambas algas. Por lo anterior se postula una interacción positiva entre el CCN y los cultivadores de pelillo del humedal marino de bahía Caulín, basada en el consumo de las frondas de lamilla que crecen en los sistemas de cultivo. De esta forma se generan nuevos temas de investigación para el estudio del potencial servicio ambiental que brinda el CCN a los cultivadores de pelillo, a través del consumo de las lamillas asentadas en sus sistemas de cultivo y los efectos sobre el rendimiento del alga cultivada.

\section{AGRADECIMIENTOS}

El trabajo fue financiado por el proyecto Núcleo de Investigación BIODES, otorgado por la Dirección de Investigación de la Universidad de Los Lagos. A Bárbara Ávila, Sebastián Rosenfeld y Dr. Roberto Quevedo por su apoyo en los análisis. Al Dr. Roberto Schlatter, Dr. Paulo Corti y Víctor Raimilla por facilitar literatura relevante. A la Dra. Paulina Gebauer y Dr. Daniel Varela por su ayuda en el diseño del estudio. A Javiera Abarzúa por confeccionar la Figura 3. A dos revisores anónimos de Gayana.

\section{BIBLIOGRAFÍA}

Altmann, J. 1973. Observational study of behavior: sampling methods. Behavior 49:227-267.

Andres, B.A., Johnson, J.A., Valenzuela, J., Morrison, R.I., Espinosa, L.A. \& Ross, R.K. 2009. Estimating eastern pacific coast populations of Whimbrels and Hudsonian Godwits, with an emphasis on Chiloé Island, Chile. Waterbirds 32:216-224.

BirdLife International. 2012. Species factsheet: Cygnus melancoryphus. URL: http://www.birdlife.org. Accesado: Octubre 10, 2014).

Bortolus, A., Iribarne, O.O. \& Martinez, M.M. 1998. Relationship between waterfowl and the seagrass Ruppia maritima in a southwestern Atlantic coastal lagoon. Estuaries 21:170717.

Briones, G. 1998. Métodos y técnicas de investigación para las ciencias sociales. Editorial Trillas, México. 368 pp.

Cáceres, C. \& Ojeda, F.P. 2000. Patrones de forrajeo en dos especies de peces intermareales herbívoros de la costa de Chile: Efecto de la abundancia y composición química del alimento. Revista Chilena de Historia Natural 73:253-260.

CORTI, P. 1996. Conducta de alimentación y capacidad de forrajeo del cisne de cuello negro (Cygnus melanocorypha Molina, 1782) en humedales de Valdivia. Tesis. Universidad Austral de Chile. Valdivia, Chile.

Corti, P. \& Schlatter, R.P. 2002. Feeding of the Black-necked Swan Cygnus melancoryphus in two wetlands of Southern Chile. Studies on Neotropical Fauna and Environment 37:9-14.

Cursach, J.A., Rau, J.R. \& Tobar, C.N. 2010. Aves en un humedal marino del sur de Chile. Revista de Biología Marina y Oceanografía 45:441-450.

Figueroa-Fábrega, L., Galaz, J. \& Merino, C. 2006. Conocimiento y conservación del cisne de cuello negro Cygnus melancoryphus (Molina, 1782) en el humedal del río Cruces, Valdivia, Chile. Gestión Ambiental 12:77-89.

García, G.O., Favero, M. \& Mariano-Jelicich, R. 2008. Redgartered Coot Fulica armillata feeding on the grapsid crab Cyrtograpsus angulatus: advantages and disvantages of an unusual food resource. Ibis 150:110-114.

Gibbons, J, Vilina, Y.A. \& CÁRCAmo, J. 2007. Distribución y 
abundancia de cisne coscoroba (Coscoroba coscoroba), cisne de cuello negro (Cygnus melancoryphus) y del flamenco chileno (Phoenicopterus chilensis) en la región de Magallanes. Anales del Instituto de la Patagonia 35:53-58.

Hoffmann, A. \& Santelices, B. 1997. Flora marina de Chile central. Editorial Universidad Católica de Chile, Santiago. $434 \mathrm{pp}$.

Hucke-Gaete R., Ruiz, J. \& Álvarez, R. 2010. Descripción de la ecorregión Chiloense. En: Conservando el mar de Chiloé, Palena y Guaitecas (Eds. Hucke-Gaete, R., Lo Moro, P. \& J. Ruiz), pp. 26-62. Comisión Nacional del Medio Ambiente, Puerto Montt.

Huntington, H.P. 1998. Observations on the utility of the semidirective interview for documenting traditional ecological knowledge. Arctic 51:237-242.

Jaramillo, E., Schlatter, R., Contreras, H., Duarte, C., Lagos, N., Paredes, E., Ulloa, J., Valenzuela, G., Peruzzo, B. \& SILVA, R. 2007. Emigration and mortality of Black-necked swans (Cygnus melancoryphus) and disappearance of the macrophyte Egeria densa in a Ramsar wetland site of southern Chile. AMBIO 36:607-610.

Johnson, A. \& Goodall, J. 1965. The birds of Chile and adjacent regions of Argentina, Bolivia and Peru. Plantt Establecimientos Gráficos, Buenos Aires. 379 pp.

Johnson, M.K., Wofford, H. \& Pearson, H.A. 1983. Microhistological techniques for food habits analysis. U.S. Department of Agriculture. Forest Service, New Orleans. $40 \mathrm{pp}$.

LoBeL, P.S. 1981. Trophic biology of herbivorous reef fishes: alimentary $\mathrm{pH}$ and digestive capabilities. Journal of Fish Biology 19:365-397.

Markham, B. 1971. Censo invernal de cisnes y flamencos en Magallanes. Anales del Instituto de la Patagonia 2:146157.

Matus, R., Díaz-Segovia, F. \& Schmitt, F. 2010. Censos Neotropicales de Aves Acuáticas en Chile - Resultados 2009. Red de Observadores de Aves y Vida Silvestre de Chile, Santiago. 52 pp.

Mckelvey, R.W. \& Verbeek, N.A.M. 1988. Habitat use, behaviour and management of Trumpeter Swans, Cygnus buccinators, wintering at Comox, British Columbia. The Canadian Field-Naturalist 102:434-441.

Muñoz-Pedreros, A. 2004. Los humedales del río Cruces y la convención de Ramsar: un intento de protección fallido. Gestión Ambiental 10:11-26.

Ortiz, P., Rodríguez, I., Arrey, P. \& Jaramillo, A. 2009. Chile. En: Important Bird Areas Americas, Priority sites for biodiversity conservation (Eds. Devenish, C., Díaz-
Fernández, D.F., Clay, R.P., Davidson, I. \& I. YépezZabala), pp. 125-134. BirdLife International, Conservation Series $N^{\circ} 16$, Quito.

Owen, M. 1975. An assessment of fecal analysis technique in waterfowl feeding studies. Journal of Wildlife Management 39:271-279.

RAU, J. 1980. Fluctuación estacional de Cygnus melanocoryphus (Molina) en Puerto Natales (Última Esperanza, XII Región de Magallanes) (Anseriformes, Anatidae). Noticiario Mensual del Museo Nacional de Historia Natural 3-4:279-280.

Sabat, P., Maldonado, K., Fariña, J.M. \& Martínez del Río, C. 2006. Osmoregulatory capacity and the ability to use marine food sources in two coastal songbirds (Cinclodes: Furnariidae) along a latitudinal gradient. Oecologia 148:250-257.

SAG. 2011. La Ley de Caza y su reglamento. Servicio Agrícola y Ganadero, Ministerio de Agricultura, Santiago. 97 pp.

Schlatter, R.P. 2005. Distribución del cisne de cuello negro en Chile y su dependencia de hábitats acuáticos de la Cordillera de la Costa. En: Historia, biodiversidad y ecología de los bosques costeros de Chile (Eds. SmithRamírez, C., Armesto, J.J. \& C. Valdovinos), pp. 498-504. Editorial Universitaria, Santiago.

Schlatter, R., Salazar, J., Villa, A. \& Meza, J. 1991a. Reproductive biology of Black-necked swan Cygnus melancoryphus at three Chilean wetland areas and feeding ecology at Rio Cruces. Wildfowl, Special Supplement 1:268-271.

Schlatter, R.P., Salazar, J. \& Meza, J. 1991b. Demography of Black-necked Swans Cygnus melancoryphus in three Chilean wetland areas. Wildfowl 1:88-94.

Schlatter, R.P. \& Simeone, A. 1999. Estado del conocimiento y conservación de las aves en mares chilenos. Estudios Oceanológicos 18:25-33.

Schlatter, R.P., Navarro, R.A. \& Corti, P. 2002. Effects of El Nino Southern Oscillation on numbers of Black-Necked Swans at Rio Cruces Sanctuary, Chile. Waterbirds 25:114-122.

Scott, P. \& The Wildfowl Trust. 1972. The Swans. Houghton Mifflin Company, Boston. 242 pp.

Vuilleumier, F. 1997. A large concentration of swans (Cygnus melancoryphus and Coscoroba coscoroba) and other waterbirds at Puerto Natales, Magallanes, Chilean Patagonia, and its significance for swans and waterfowl conservation. Ornitología Neotropical 8:1-5.

ZAR J.H. 1999. Biostatistical Analysis. Englewood Cliffs. Prentice Hall, New Jersey. 663 pp.

Recibido: 16.01 .15

Aceptado: 14.08.15 